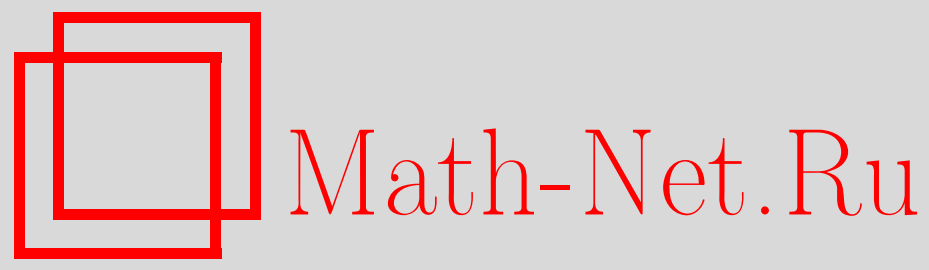

С. В. Савченко, Специальные потоки, построенные по счетным топологическим цепям Маркова, Функи. анализ и его прил., 1998, том 32, выпуск 1, 40-53

DOI: https://doi.org/10.4213/faa396

Использование Общероссийского математического портала MathNet.Ru подразумевает, что вы прочитали и согласны с пользовательским соглашением http://www . mathnet.ru/rus/agreement

Параметры загрузки:

IP : 35.174 .16 .151

26 апреля 2023 г., $11: 38: 29$

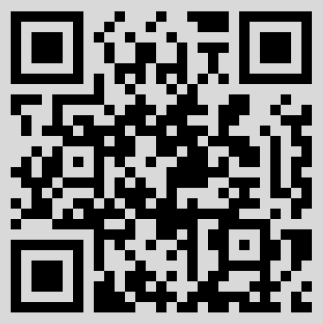


Функииональный анализ и его приложения

1998, т. 32, вып. 1, с. 40-53

Удк 517.987.5+517.938

\title{
Специальные потоки, построенные по счетным топологическим цепям Маркова
}

\author{
(C) 1998. C. В. Савченко
}

\section{§1. Введение}

Пусть $T$ - гомеоморфизм топологического пространства $Z$ и $f$ - неотрицательная непрерывная на $Z$ функция, такая, что совокупность ее нулей $J(f)$ является подмножеством множества $\{z \in Z: T z=z\}$. Динамической системе $(Z, T)$ и функции $f$ сопоставим пространство $Z_{f}=\{(z, u): z \in Z$, $0 \leqslant u \leqslant f(z)\}$, в котором отождествляются точки $(z, f(z))$ и $(T z, 0)$. При $0 \leqslant u, u+t \leqslant f(z)$ положим $T_{f}^{t}(z, u)=(z, u+t)$. Если для любого $z \in Z \backslash J(f)$ выполняется условие

$$
\sum_{m=1}^{\infty} f\left(T^{m} z\right)=\sum_{m=1}^{\infty} f\left(T^{-m} z\right)=\infty,
$$

то для остальных $t \in \mathbb{R}$ отображение $T_{f}^{t}$ определим, используя идентификацию указанных точек и исходя из того, что $\left\{T_{f}^{t}\right\}-$ однопараметрическая группа преобразований. Для такого $z$, что $f(z)=0$, положим $T_{f}^{t}(z, 0)=$ $(z, 0)$ при любом $t \in \mathbb{R}$. Семейство преобразований $T_{f}=\left\{T_{f}^{t}\right\}$ называется специальныл потоком, построенным по динамической системе $(Z, T)$ и функции $f$, а точки $(z, 0)$, где $z \in J(f)$, 一 неподвижными точками потока $T_{f}$.

Пусть $\hat{I}\left(Z_{f}\right)$ - множество всех инвариантных эргодических относительно $T_{f}$ вероятностных мер на $Z_{f}$. Обозначим через $h\left(T_{f}^{t}, \nu\right)$ энтропию преобразования $T_{f}^{t}$ относительно меры $\nu \in \hat{I}\left(Z_{f}\right)$. По определению энтропия $h\left(T_{f}, \nu\right)$ потока $T_{f}$ относительно меры $\nu$ равна $h\left(T_{f}^{1}, \nu\right)$. Положим

$$
h\left(T_{f}\right)=\sup \left\{h\left(T_{f}, \nu\right): \nu \in \hat{I}\left(Z_{f}\right)\right\} .
$$

Пусть $\hat{I}_{f}^{\infty}(Z)$ - множество эргодических относительно $T$ мер $\mu$ на $Z$ (необязательно конечных) с $\int f d \mu<\infty$. Положим

$$
\mu_{f}=\frac{\left.(\mu \times m)\right|_{Z_{f}}}{\int f d \mu}
$$

где $m$ - мера Лебега на действительной прямой $\mathbb{R}$, а $\left.(\mu \times m)\right|_{Z_{f}}$ означает ограничение меры $\mu \times m$ на множество $Z_{f}$ (если $\mu$ сосредоточена в точке

\footnotetext{
* Работа выполнена при финансовой поддержке RFBR (грант INTAS-RFBR 95-418).
} 
$z \in J(f)$, то будем считать, что $\mu_{f}$ - вероятностная мера, сосредоточенная в точке $(z, 0))$. Нетрудно проверить, что $\hat{I}\left(Z_{f}\right)=\left\{\mu_{f}: \mu \in \hat{I}_{f}^{\infty}(Z)\right\}$. Пусть $\hat{I}_{f}(Z)$ - множество всех эргодических относительно преобразования $T$ вероятностных мер на $Z$ с $\int f d \mu<\infty$. Для любой непрерывной неотрицательной на $Z$ функции $f$ и меры $\mu \in \hat{I}_{f}(Z)$ положим

$$
P(Z ; f ; \mu)=h(T, \mu)-\int_{Z} f d \mu,
$$

где $h(T, \mu)$ - энтропия преобразования $T$ относительно меры $\mu$,

$$
\begin{aligned}
& P(Z ; f)=\sup \left\{P(Z ; f ; \mu): \mu \in \hat{I}_{f}(Z)\right\} \\
& \hat{I}(Z ; f)=\left\{\mu \in \hat{I}_{f}(Z): P(Z ; f ; \mu)=P(Z ; f)\right\} .
\end{aligned}
$$

В случае когда функция $f$ отделена от нуля, т.е. $\inf \{f(z): z \in Z\}>0$ (например, множество $Z$ является компактом, а $f$ - непрерывной положительной функцией), любая мера $\nu \in \hat{I}\left(Z_{f}\right)$ имеет вид $\mu_{f}$, где $\mu \in \hat{I}_{f}(Z)$. Если при этом $P(Z ; 0)<\infty$, то, используя формулу Абрамова [1] для метрической энтропии потока, нетрудно проверить, что величина $h\left(T_{f}\right)$ является единственным решением уравнения $P(Z ; h f)=0$ (см. [2, Prop. 6.1]). В эргодической теории особый интерес представляют меры с максимальной энтропией, т. е. меры $\nu \in \hat{I}\left(Z_{f}\right)$, для которых $h\left(T_{f}, \nu\right)=h\left(T_{f}\right)$. Обозначим через $\hat{I}\left(Z_{f} ; 0\right)$ множество всех таких мер. Очевидно, что в нашем случае $\hat{I}\left(Z_{f} ; 0\right)=\left\{\mu_{f}: \mu \in \hat{I}\left(Z ; h\left(T_{f}\right) f\right)\right\}$ (cм. [2, Prop. 6.1]).

Если функция $f$ не отделена от нуля, то могут существовать и бесконечные меры $\mu$ на $Z$, для которых $\mu_{f} \in \hat{I}\left(Z_{f}\right)$. Поэтому необходимо обобщить формулу Абрамова на случай такой меры. Это сделано в $\S 2$ данной работы, причем в качестве величины $h(T, \mu)$ выбрана энтропия Кренгеля [3]. Обобщенная формула Абрамова - единственный результат статьи, относящийся к абстрактному специальному потоку; остальная часть статьи посвящена специальным потокам, построенным по топологическим цепям Маркова (сокращено ТМЦ). Они возникают при изучении гиперболических потоков на компактных многообразиях, удовлетворяющих аксиоме А Смейла [4]. Случай специального потока, построенного по конечной ТМЦ и гёльдеровской относительно экспоненциальной метрики функции (такой поток называется гиперболическим символическим потоком), был подробно рассмотрен Боуэном и Рюэлем в работе [5]. Специальные потоки над счетными ТМЦ возникают при изучении рассеивающих биллиардов (см. [6]). В дополнении к книге [7] ее автором высказано предположение о том, что для некомпактных многообразий постоянной отрицательной кривизны с конечным объемом, например для модулярной поверхности, существуют счетные марковские разбиения и, следовательно, соответствующие геодезические потоки также исследуются при помощи специальных потоков, построенных по счетным ТМЩ. О таких потоках известно намного меньше по сравнению с гиперболическими (это, в частности, связано с тем, что в счетном случае для гёльдеровской функции до сих пор не изучена структура множества $\left.\hat{I}_{f}(Z)\right)$. В настоящей 
работе мы исследуем потоки, построенные по счетным ТМЦ и положительным локально постоянным функциям. При помощи формулы Кренгеля в 3 вычислена топологическая энтропия и получены необходимые и достаточные условия существования (и единственности) меры с максимальной энтропией. Оказывается, что игнорирование бесконечных мер не сказывается на величине топологической энтропии таких символических потоков, однако при этом мы можем «потерять» меры, на которых достигается максимум энтропии. Вопрос, насколько общей является эта ситуация, остается открытым.

Помимо мер с максимальной энтропией большой интерес представляют законы распределения числа периодических траекторий потоков (в последнее время это объясняется, в первую очередь, задачами квантового хаоса). Для гиперболического символического потока мероморфное продолжение дзетафункции Рюэля-Смейла (эта функция является аналогом дзета-функции Римана - роль простых чисел здесь играют экспоненты от наименьших периодов замкнутых орбит) было построено Рюэлем в [8]. Это позволило Пэрри и Полликоту (см. [9]) при помощи тауберовой теоремы Икехары-Винера получить закон Хубера (см. [10]) распределения числа периодических траекторий, аналогичный асимптотическому закону Чебышёва в теории простых чисел. Для случая счетной ТМЦ и положительной локально постоянной функции аналитические свойства дзета-функции Рюэля-Смейла и возможные законы распределения периодических траекторий были изучены в работе [11] автора (см. 6 ). Из результатов параграфов $\S 2$ и 3 данной статьи, в которых развиваются идеи, изложенные в замечании 18 из [11], следует, что существование мероморфного продолжения для дзета-функции и справедливость закона Хубера зависят от устойчивости структуры множества мер с максимальной энтропией относительно малых возмущений функции $f$ (см. следствие 1 ).

В теории динамических систем на компактных метрических пространствах обычно рассматривают потоки, разделяющие траектории (определение см. в [12]). Неподвижные точки таких потоков являются изолированными. Если отказаться от этого условия, т.е. разрешить функции $f$ обращаться в нуль, то уже в случае конечной ТМЦ можно получить принципиально новые результаты о множестве максимальных мер и законах распределения периодических траекторий. В $\S 4$ в качестве такой функции $f$ с $\operatorname{card} J(f)=1$ мы рассматриваем функции из класса $\mathscr{F}(X(G) ; \mathbf{v})$ (здесь $X(G)$ - фазовое пространство ТМЦ, а $\mathbf{v}$ - некоторый фиксированный символ), введенного в работе [13] (в [13] исследуются потоки, построенные по положительным функциям из этого класса). Оказывается, что в этом случае для соответствующего специального потока мера с максимальной энтропией не обязана существовать или она может иметь вид $\mu_{f}$, где $\mu(X(G))=\infty$.

Автор надеется, что полученные в работе результаты окажутся полезными в понимании принципиальных трудностей, возникающих в пока мало исследованных рассеивающих биллиардах с нулевыми углами в точках излома границы.

\section{§2. Обобщенная формула Абрамова}

Пусть $T$ - автоморфизм пространства $Z$ с сигма-алгеброй $\Xi$ и сигмаконечной инвариантной мерой $\mu$. Мера $\mu$ называется эргодической (относительно $T)$, если $\mu(E)=0$ или $\mu(Z \backslash E)=0$ для любого инвариантного 
множества $E \in \Xi$. Множество $E \subseteq Z$ называется блуждаюшим (относительно $T$ ), если множества $E, T^{-1} E, \ldots, T^{-n} E, \ldots$ попарно не пересекаются. Сигма-конечная мера $\mu$ называется консервативной относительно $T$, если $\mu(E)=0$ для любого блуждающего множества $E$. В случае когда имеется блуждающее множество $E$ с $\mu(E)>0$, для любой положительной функции $f$ с конечным интегралом Лебега найдется такое множество $B_{f} \in \Xi$ с $\mu\left(B_{f}\right)>0$, что для почти каждого $z \in B_{f}$ справедливо неравенство $\sum_{m=1}^{\infty} f\left(T^{m} z\right)<\infty$ (см. [14]).

Если мера сосредоточена в неподвижной относительно потока точке, то ее энтропия равна нулю. Для того чтобы вычислить величину $h\left(T_{f}, \mu_{f}\right)$ для произвольной меры $\mu \in \hat{I}_{f}^{\infty}(Z)$, воспользуемся энтропией Кренгеля, к определению которой мы и приступаем.

Пусть $E \in \Xi$. Обозначим через $E^{\infty}$ множество всех $z \in Z$, для которых $T^{n} z \in E$ при бесконечно многих как положительных, так и отрицательных $n$. Положим $E_{\text {inf }}=E \cap E^{\infty}$.

В случае консервативного автоморфизма $T$ имеем $\mu\left(E \backslash E_{\text {inf }}\right)=0$ (см. [15]). Поэтому можно определить производный автоморфизм $\left.T\right|_{E}$ пространства $E$ с сигма-алгеброй $\left.\Xi\right|_{E}=\{B \cap E: B \in \Xi\}$ и мерой $\left.\mu\right|_{E}$, где $\left.\mu\right|_{E}(B)=\mu(B \cap E)$, по следующему правилу:

$$
\left.T\right|_{E} z= \begin{cases}T^{r_{E}(z)} z & \text { при } z \in E_{\mathrm{inf}}, \text { где } r_{E}(z)=\min \left\{n \geqslant 1: T^{n} z \in E\right\}, \\ z & \text { при } z \notin E_{\mathrm{inf}} .\end{cases}
$$

Определение энтропии $h(T, \mu)$ для вероятностной инвариантной меры $\mu$ хорошо известно (см. $[16,17])$. Точно также можно определить величину $h(T, \mu)$ и для произвольной конечной инвариантной меры $\mu$ (подробности см. в $\S 3$ работы [3]). Обозначим через $\bar{\mu}$ нормированную меру $(1 / \mu(Z)) \mu$. Пользуясь определением энтропии для конечной меры $\mu$, легко проверить, что

$$
h(T, \mu)=h(T, \bar{\mu}) \mu(Z) .
$$

Для произвольной консервативной меры $\mu$ определим величину $h(T, \mu)[3]$, положив

$$
h(T, \mu)=\sup \left\{h\left(\left.T\right|_{E},\left.\mu\right|_{E}\right): E \subseteq Z, 0<\mu(E)<\infty\right\} .
$$

Множество $E$ будем называть заметающим (sweep-out-set), если

$$
\mu\left(Z \backslash \bigcup_{n=0}^{\infty} T^{-n} E\right)=0
$$

Для эргодического автоморфизма любое множество положительной меры является заметающим. В силу теоремы 3.1 из [3] для любого заметающего множества $E$ конечной меры $h(T, \mu)=h\left(\left.T\right|_{E},\left.\mu\right|_{E}\right)=h\left(\left.T\right|_{E}, \overline{\left.\mu\right|_{E}}\right) \mu(E)$ (если $\mu(Z)=1$, то эта формула является утверждением известной теоремы Абрамова об энтропии производного автоморфизма; см. [18]).

Teоpema 1. Пусmь $\mu \in \hat{I}_{f}^{\infty}(Z)$. Тогдa

$$
h\left(T_{f}, \mu_{f}\right)=\frac{h(T, \mu)}{\int f d \mu} .
$$


ДокАЗАТЕЛЬСТво. Пусть $Z^{h}=\{z \in Z: f(z)>h\}$. Выберем такое $h>0$, при котором $\mu\left(Z^{h}\right)>0$. Так как по условию $\int f d \mu<\infty$, то в силу неравенства Чебышёва имеем $\mu\left(Z^{h}\right)<\infty$. Производный автоморфизм $\left.T_{f}^{h}\right|_{Z^{h} \times[0, h)}$, индуцированный автоморфизмом $T_{f}^{h}$ на множестве $Z^{h} \times[0, h)$, определяется формулой

$$
\left.T_{f}^{h}\right|_{Z^{h} \times[0, h)}(z, u)=\left(\left.T\right|_{Z^{h}} z,\left(u-\sum_{k=1}^{r_{Z^{h}}(z)} f\left(T^{k-1} z\right)\right)(\bmod h)\right) .
$$

Очевидно, что $\overline{\left.\mu_{f}\right|_{Z^{h} \times[0, h)}}=\overline{\left.\mu\right|_{Z^{h}}} \times \overline{\left.m\right|_{[0, h)}}$. Автоморфизм $\left.T_{f}^{h}\right|_{Z^{h} \times[0, h)}$ пространства $Z^{h} \times[0, h)$ с вероятностной мерой $\overline{\left.\mu\right|_{Z^{h}}} \times \overline{\left.m\right|_{[0, h)}}$ метрически изоморфен автоморфизму $\left.T_{f / h}^{1}\right|_{Z^{h} \times[0,1)}$ пространства $Z^{h} \times[0,1)$ с вероятностной мерой $\overline{\left.\mu\right|_{Z^{h}}} \times\left. m\right|_{[0,1)}$ :

$$
\left.T_{f / h}^{1}\right|_{Z^{h} \times[0,1)}(z, u)=\left(\left.T\right|_{Z^{h}} z,\left(u-\frac{1}{h} \sum_{k=1}^{r_{Z^{h}}(z)} f\left(T^{k-1} z\right)\right)(\bmod 1)\right)
$$

(изоморфизм «осушествляет» отображение $\psi:(z, u) \rightarrow(z, u / h))$. В силу леммы 1 работы [1] имеем

$h\left(\left.T_{f}^{h}\right|_{Z^{h} \times[0, h)}, \overline{\left.\mu\right|_{Z^{h}}} \times \overline{\left.m\right|_{[0, h)}}\right)=h\left(\left.T_{f / h}^{1}\right|_{Z_{h} \times[0,1)}, \overline{\left.\mu\right|_{Z^{h}}} \times\left. m\right|_{[0,1)}\right)=h\left(\left.T\right|_{Z^{h}}, \overline{\left.\mu\right|_{Z^{h}}}\right)$.

По теореме Абрамова об энтропии производного автоморфизма [18]

$$
\begin{aligned}
h\left(T_{f}^{h}, \mu_{f}\right) & =h\left(\left.T_{f}^{h}\right|_{Z^{h} \times[0, h)}, \overline{\left.\mu\right|_{Z^{h}}} \times \overline{\left.m\right|_{[0, h)}}\right) \mu_{f}\left(Z^{h} \times[0, h)\right) \\
& =\frac{h\left(\left.T\right|_{Z^{h}}, \overline{\left.\mu\right|_{Z^{h}}}\right) \cdot \mu\left(Z^{h}\right) \cdot h}{\int f d \mu}=\frac{h}{\int f d \mu} h\left(\left.T\right|_{Z^{h}},\left.\mu\right|_{Z^{h}}\right)=\frac{h}{\int f d \mu} h(T, \mu)
\end{aligned}
$$

и, таким образом,

$$
h\left(T_{f}, \mu_{f}\right)=h\left(T_{f}^{1}, \mu_{f}\right)=\frac{h\left(T_{f}^{h}, \mu_{f}\right)}{h}=\frac{h(T, \mu)}{\int f d \mu} .
$$

Теорема доказана.

\section{§3. Специальные потоки, построенные по счетным ТМЦ и локально постоянным функциям}

В оставшейся части статьи в качестве динамической системы $(Z, T)$ мы будем рассматривать преобразование сдвига $\sigma$ на пространстве $X(G)$ двусторонне бесконечных последовательностей $x=\left\{x_{i}\right\}$ символов из множества $\Omega$, соответствуюших путям счетного ориентированного связного графа $G$ с множеством вершин $V(G)=\Omega$. Такая динамическая система называется топологической марковской цепью (сокращенно ТМЦ). Функция $f$ в этом параграфе будет предполагаться зависящей только от координаты $x_{0}$ точки $x=\left\{x_{i}\right\}$ пространства $X(G)$ (класс таких функций мы будем обозначать 
через $B^{0}(X(G))$ ), положительной и удовлетворяющей условию (1) (семейство таких функций обозначим через $\left.B_{+}^{0}(X(G))\right)$.

Любому конечному пути $\gamma=\left\{\mathbf{v}_{i}\right\}_{i=0}^{k}$, где $k \geqslant 0$ и $\mathbf{v}_{i} \in V(G)$ при $i=$ $0, \ldots, k$, графа $G$ длины $\ell(\gamma)$, равной по определению $k$, сопоставим цилиндрическое множество $B(\gamma)=\left\{x \in X(G): x_{i}=\mathbf{v}_{i}, i=0, \ldots, \ell(\gamma)\right\}$. Если путь $\gamma$ состоит из единственной вершины $\mathbf{v}$ (в этом случае $\ell(\gamma)=0$ ), то будем писать $B(\mathbf{v})$ вместо $B(\gamma)$. Для любого пути $\gamma$ с $\ell(\gamma) \geqslant 1$ положим

$$
f^{*}(\gamma)=\sum_{m=0}^{\ell(\gamma)-1} f\left(\sigma^{m} x\right), \quad \text { где } x \in B(\gamma)
$$

(очевидно, что при $f \in B^{0}(X(G))$ значение определяемой величины не зависит от выбора точки в множестве $B(\gamma))$.

Путь $\gamma \quad \mathbf{c} \quad \mathbf{v}_{0}=\mathbf{v}_{\ell(\gamma)}=\mathbf{v}$ будем называть $\mathbf{v}$-циклом. Обозначим через $K(G ; \mathbf{v})$ множество всех $\mathbf{v}$-циклов в графе $G$, а через $L(G ; \mathbf{v})$ - множество тех $\mathbf{v}$-циклов, у которых $\mathbf{v}_{i} \neq \mathbf{v}$ при $1 \leqslant i \leqslant \ell(\gamma)-1$ (если существует $\mathbf{v}$-цикл $\gamma \mathbf{c} \ell(\gamma)=1$, то будем считать, что он также принадлежит множеству $L(G ; \mathbf{v}))$.

Предположим, что $\mu$ - консервативная эргодическая относительно $\sigma$ мера и $0<\mu(B(\mathbf{v}))<\infty$. Обозначим через $B^{\infty}(\mathbf{v})$ множество всех тех $x \in X(G)$, у которых $x_{i}=\mathbf{v}$ при бесконечно многих как положительных, так и отрицательных $i$. Так как $\mu\left(X(G) \backslash B^{\infty}(\mathbf{v})\right)=0$, то множества $\sigma^{k} B(\gamma)$, где $\gamma \in L(G ; \mathbf{v}), 0 \leqslant k \leqslant \ell(\gamma)-1$, образуют разбиение пространства $X(G)$ с мерой $\mu$. Поэтому

$$
\int f d \mu=\sum_{\gamma \in L(G ; \mathbf{v})} \sum_{k=0}^{\ell(\gamma)-1} f\left(\sigma^{k} B(\gamma)\right) \mu\left(\sigma^{k} B(\gamma)\right)=\sum_{\gamma \in L(G ; \mathbf{v})} f^{*}(\gamma) \mu(B(\gamma))
$$

(здесь через $f\left(\sigma^{k} B(\gamma)\right)$ мы обозначили величину $f\left(\sigma^{k} x\right)$, где $x \in B(\gamma)$ ). Положим

$$
H(\mu, \mathbf{v})=\sum_{\gamma \in L(G ; \mathbf{v})}-\overline{\left.\mu\right|_{B(\mathbf{v})}}(B(\gamma)) \ln \overline{\left.\mu\right|_{B(\mathbf{v})}}(B(\gamma))
$$

1. Топологическая энтропия. Для того чтобы получить выражение, связывающее топологическую энтропию и множество нулей функции $P(X(G) ; \varepsilon f)$, нам понадобится следующая лемма, смысл которой состоит в том, что величина $h\left(\sigma_{f}\right)$ не изменится, если в ее определении вместо множества всех эргодических вероятностных мер взять только меры вида $\mu_{f}$, где $\mu \in \hat{I}_{f}(X(G))$.

Лемма 1. Пусть $f \in B_{+}^{0}(X(G))$. Тогда

$$
h\left(\sigma_{f}\right)=\sup \left\{h\left(\sigma_{f}, \mu_{f}\right): \mu \in \hat{I}_{f}(X(G))\right\} .
$$

ДокАЗАТЕльство. Пусть $h\left(\sigma_{f}\right)<\infty$. Тогда для любого $\varepsilon>0$ существует такая мера $\mu \in \hat{I}_{f}^{\infty}(X(G))$, что $h\left(\sigma_{f}, \mu_{f}\right)>h\left(\sigma_{f}\right)-\varepsilon$. Выберем $\mathbf{v} \in V(G)$, для которого $\mu(B(\mathbf{v}))>0$. Так как $\mu(B(\mathbf{v}))<\infty$ (это следует из условий 
$f \in B_{+}^{0}(X(G))$ и $\left.\int f d \mu<\infty\right)$ и $(\lambda \mu)_{f}=\mu_{f}$ при любом $\lambda \in(0, \infty)$, то без ограничения общности можно считать, что $\mu(B(\mathbf{v}))=1$.

Занумеруем элементы множества $L(G ; \mathbf{v})$ произвольным образом и положим $q_{n}=\sum_{i=1}^{n} \mu\left(B\left(\gamma_{i}\right)\right)$. Очевидно, что $\lim _{n \rightarrow \infty} q_{n}=\mu(B(\mathbf{v}))=1$.

Обозначим через $\mu^{n}$ инвариантную меру, для которой $\mu^{n}\left(X(G) \backslash B^{\infty}(\mathbf{v})\right)$ $=0, \mu^{n}\left(B\left(\gamma_{i}\right)\right)=\mu\left(B\left(\gamma_{i}\right)\right) / q_{n}, i=1, \ldots, n, \mu^{n}\left(B\left(\gamma_{i}\right)\right)=0$ при $i>n$ и мера $\left.\mu^{n}\right|_{B(\mathbf{v})}$ является бернуллиевской, т. е. разбиения $\left(\left.\sigma\right|_{B(\mathbf{v})}\right)^{p} \beta(\mathbf{v})$, где $p \in \mathbb{Z}$ и $\beta(\mathbf{v})$ - разбиение множества $B(\mathbf{v}) \cap B^{\infty}(\mathbf{v})$ на множества $B(\gamma) \cap B^{\infty}(\mathbf{v})$, $\gamma \in L(G ; \mathbf{v})$, независимы относительно меры $\left.\mu^{n}\right|_{B(\mathbf{v})}$. Очевидно, что

$$
\mu^{n}(X(G))=\frac{1}{q_{n}} \sum_{i=1}^{n} \ell\left(\gamma_{i}\right) \mu\left(B\left(\gamma_{i}\right)\right)<\infty
$$

(см. (4) при $f=1$ и $\mu=\mu^{n}$ ). Нетрудно проверить (см. (4) и (5)), что

$$
\begin{aligned}
h\left(\sigma, \mu^{n}\right) & =h\left(\left.\sigma\right|_{B(\mathbf{v})},\left.\mu^{n}\right|_{B(\mathbf{v})}\right)=H\left(\mu^{n}, \mathbf{v}\right) \\
& =-\frac{1}{q_{n}} \sum_{i=1}^{n} \mu\left(B\left(\gamma_{i}\right)\right) \ln \mu\left(B\left(\gamma_{i}\right)\right)+\ln q_{n}, \\
\int f d \mu^{n} & =\frac{1}{q_{n}} \sum_{i=1}^{n} f^{*}\left(\gamma_{i}\right) \mu\left(B\left(\gamma_{i}\right)\right) .
\end{aligned}
$$

Поэтому

$$
\lim _{n \rightarrow \infty} h\left(\sigma, \mu^{n}\right)=H(\mu, \mathbf{v}) \quad \text { и } \quad \lim _{n \rightarrow \infty} \int f d \mu^{n}=\int f d \mu<\infty .
$$

Предположим, что $H(\mu, \mathbf{v})=\infty$. Тогда $\lim _{n \rightarrow \infty} h\left(\sigma, \mu^{n}\right)=\infty$ и, следовательно, $\lim _{n \rightarrow \infty} h\left(\sigma_{f}, \mu_{f}^{n}\right)=\infty$, что противоречит условию $h\left(\sigma_{f}\right)<\infty$. Поэтому $H(\mu, \mathbf{v})<\infty$. Выберем $n$ так, чтобы

$$
\frac{H(\mu, \mathbf{v})}{\int f d \mu}-\frac{h\left(\sigma, \mu^{n}\right)}{\int f d \mu^{n}}<\varepsilon .
$$

Так как $h(\sigma, \mu)=h\left(\left.\sigma\right|_{B(\mathbf{v})},\left.\mu\right|_{B(\mathbf{v})}\right) \leqslant H(\mu, \mathbf{v})$, то $h\left(\sigma_{f}, \mu_{f}\right)-h\left(\sigma_{f}, \mu_{f}^{n}\right)<\varepsilon$. Поэтому справедливо неравенство $h\left(\sigma_{f}, \mu_{f}^{n}\right)>h\left(\sigma_{f}\right)-2 \varepsilon$. Отсюда и из того, что $\varepsilon>0$ произвольно, следует, что

$$
h\left(\sigma_{f}\right)=\sup \left\{h\left(\sigma_{f}, \mu_{f}\right): \mu \in \hat{I}_{f}^{\infty}(X(G)), \mu(X(G))<\infty\right\} .
$$

Очевидно, что $\left\{\mu_{f}: \mu \in \hat{I}_{f}^{\infty}(X(G)), \mu(X(G))<\infty\right\}=\left\{\mu_{f}: \mu \in \hat{I}_{f}(X(G))\right\}$ (напомним, что $(\lambda \mu)_{f}=\mu_{f}$ при любом $\lambda \in(0, \infty)$ ). Поэтому равенство $(6)$ справедливо, если $h\left(\sigma_{f}\right)<\infty$. Случай $h\left(\sigma_{f}\right)=\infty$ разбирается аналогичным образом. Лемма доказана.

Положим

$$
h(f)=\left\{\begin{array}{l}
\infty, \text { если } P(X(G) ; h f)>0 \text { для любого } h \in \mathbb{R}, \\
\inf \{h: P(X(G) ; h f) \leqslant 0\} \text { в противном случае. }
\end{array}\right.
$$


Пусть $\mathbf{r}_{\mathbf{v}}(f)$ - радиус сходимости ряда

$$
\sum_{\gamma \in L(G ; \mathbf{v})} \exp \left(-f^{*}(\gamma)\right) z^{\ell(\gamma)}
$$

Если при $z=\mathbf{r}_{\mathbf{v}}(f)$ сумма ряда $(7)$ меньше единицы, то $\exp (-P(X(G) ; f))$ $=\mathbf{r}_{\mathbf{v}}(f)$. В противном случае величина $\exp (-P(X(G) ; f))$ совпадает с единственным решением на промежутке $[0, \infty)$ уравнения $\varphi_{X(G), B(\mathbf{v}), f}(z)=1$, где $\varphi_{X(G), B(\mathbf{v}), f}(z)$ - сумма ряда $(7)$ (см. [19]). Используя все эти замечания, теперь нетрудно показать, что при любом $\mathbf{v} \in V(G)$ справедливо равенство

$$
h(f)=\inf \left\{h: \sum_{\gamma \in L(G ; \mathbf{v})} \exp \left(-h f^{*}(\gamma)\right) \leqslant 1\right\} .
$$

TeOpema 2. Пycmb $f \in B_{+}^{0}(X(G))$. Тогдa

$$
h\left(\sigma_{f}\right)=h(f) .
$$

ДокАЗАТЕльСтво. Предположим, что $h\left(\sigma_{f}\right)<h(f)$. Тогда справедливо неравенство $P\left(X(G) ; h\left(\sigma_{f}\right) f\right)>0$ и, следовательно, существует мера $\mu \in$ $\hat{I}_{f}(X(G))$, для которой $h(\sigma, \mu)-h\left(\sigma_{f}\right) \int f d \mu>0$. В силу формулы Абрамова $h\left(\sigma_{f}, \mu_{f}\right)=h(\sigma, \mu) / \int f d \mu>h\left(\sigma_{f}\right)$, что противоречит определению величины $h\left(\sigma_{f}\right)$. Поэтому $h\left(\sigma_{f}\right) \geqslant h(f)$.

Покажем теперь, что величина $h\left(\sigma_{f}\right)$ не больше любого $h$, для которого $P(X(G) ; h f) \leqslant 0$. Действительно, в этом случае при любом $\mu \in \hat{I}_{f}(X(G))$ имеем $h(\sigma, \mu)-h \int f d \mu \leqslant 0$ и, следовательно, в силу формулы Абрамова $h\left(\sigma_{f}, \mu_{f}\right)$ $\leqslant h$. Из леммы 1 получаем $h\left(\sigma_{f}\right) \leqslant h$. Таким образом, $h\left(\sigma_{f}\right) \leqslant h(f)$. Теорема доказана.

2. Меры с максимальной энтропией. Случай $h\left(\sigma_{f}\right)<\infty$. В 11 динамической системе $(Z, T)$ и функции $f$ мы сопоставили пространство $Z_{f}$. В случае когда $Z=X(G)$ и $T=\sigma$, условимся писать $X_{f}(G)$ вместо $X(G)_{f}$.

TeOpema 3. Пусть $f \in B_{+}^{0}(X(G))$ u $h\left(\sigma_{f}\right)<\infty$. Тогда или $\hat{I}\left(X_{f}(G) ; 0\right)$ $=\varnothing$, или $\operatorname{card} \hat{I}\left(X_{f}(G) ; 0\right)=1$. Для существования мерьл с максимальной энтропией относительно потока $\sigma_{f}$ необходимо и достаточно, итобь $n р и$ некотором (а следовательно, и при любом) v $\in V(G)$ вылолянялись следующие условия:

$$
\begin{gathered}
\sum_{\gamma \in L(G ; \mathbf{v})} \exp \left(-h\left(\sigma_{f}\right) f^{*}(\gamma)\right)=1, \\
\sum_{\gamma \in L(G ; \mathbf{v})} f^{*}(\gamma) \exp \left(-h\left(\sigma_{f}\right) f^{*}(\gamma)\right)<\infty
\end{gathered}
$$

ДокАЗАтЕльство. Предположим, что существует мера $\mu \in \hat{I}_{f}^{\infty}(X(G))$, для которой $\mu_{f} \in \hat{I}\left(X_{f}(G) ; 0\right)$. Выберем такое $\mathbf{v} \in V(G)$, что $\mu(B(\mathbf{v}))>0$. Без ограничения общности можно предполагать величину $\mu(B(\mathbf{v}))$ равной единице. 
Мере $\mu$ сопоставим $\sigma$-инвариантную меру $\tilde{\mu}$, для которой $\tilde{\mu}\left(X(G) \backslash B^{\infty}(\mathbf{v})\right)$ $=0, \tilde{\mu}(B(\mathbf{v}))=1$, мера $\left.\tilde{\mu}\right|_{B(\mathbf{v})}$ является бернуллиевской и $\tilde{\mu}(B(\gamma))=\mu(B(\gamma))$ при всех $\gamma \in L(G ; \mathbf{v})$. Очевидно, что $h(\sigma, \tilde{\mu})=h\left(\left.\sigma\right|_{B(\mathbf{v})},\left.\tilde{\mu}\right|_{B(\mathbf{v})}\right)=H(\mu, \mathbf{v})$. Кроме того, всегда справедливо неравенство $h\left(\left.\sigma\right|_{B(\mathbf{v})},\left.\mu\right|_{B(\mathbf{v})}\right) \leqslant H(\mu, \mathbf{v})$, причем при $H(\mu, \mathbf{v})<\infty$ равенство в нем достигается тогда и только тогда, когда $\mu=\tilde{\mu}$ (см. [17]). Из (4) следует, что $\int f d \tilde{\mu}=\int f d \mu$. Таким образом, $\tilde{\mu}_{f} \in \hat{I}\left(X_{f}(G)\right)$ и в силу обобщенной формулы Абрамова $h\left(\sigma_{f}, \mu_{f}\right) \leqslant$ $h\left(\sigma_{f}, \tilde{\mu}_{f}\right)$, причем если $\mu \neq \tilde{\mu}$, то неравенство строгое.

Занумеруем элементы из $L(G ; \mathbf{v})$ произвольным образом и положим $p_{i}(\mu)$ $=\mu\left(B\left(\gamma_{i}\right)\right)$. Из всего сказанного выше следует, что $\mu=\tilde{\mu}$ и $H(\mu, \mathbf{v}) / \int f d \mu$ $=h\left(\sigma_{f}\right)$. Поэтому величины $p_{i}(\mu)$ удовлетворяют следующему соотношению:

$$
\begin{gathered}
\max \left\{-\sum_{i=1}^{\infty} p_{i}\left(h\left(\sigma_{f}\right) f^{*}\left(\gamma_{i}\right)+\ln p_{i}\right): p_{i} \geqslant 0, \sum_{i=1}^{\infty} f^{*}\left(\gamma_{i}\right) p_{i}<\infty\right\} \\
=-\sum_{i=1}^{\infty} p_{i}(\mu)\left(h\left(\sigma_{f}\right) f^{*}\left(\gamma_{i}\right)+\ln p_{i}(\mu)\right)=0 .
\end{gathered}
$$

Предположим, что $p_{m}(\mu)=0$ при некотором $m \geqslant 1$. Выберем любое $k \geqslant 1$, для которого $p_{k}(\mu)>0$. Тогда существует такое $\delta>0$, что при каждом $\varepsilon \in(0, \delta)$ справедливо неравенство

$$
\begin{gathered}
\varepsilon \ln \varepsilon+h\left(\sigma_{f}\right) f^{*}\left(\gamma_{m}\right) \varepsilon+\left(p_{k}(\mu)-\varepsilon\right) \ln \left(p_{k}(\mu)-\varepsilon\right)+h\left(\sigma_{f}\right) f^{*}\left(\gamma_{k}\right)\left(p_{k}(\mu)-\varepsilon\right) \\
<p_{k}(\mu) \ln p_{k}(\mu)+h\left(\sigma_{f}\right) f^{*}\left(\gamma_{k}\right) p_{k}(\mu) .
\end{gathered}
$$

Пусть $\mu^{\varepsilon}$ - мера, для которой $\mu^{\varepsilon}\left(X(G) \backslash B^{\infty}(\mathbf{v})\right)=0,\left.\mu^{\varepsilon}\right|_{B(\mathbf{v})}$ является мерой Бернулли, $\mu^{\varepsilon}\left(B\left(\gamma_{m}\right)\right)=\varepsilon, \mu^{\varepsilon}\left(B\left(\gamma_{k}\right)\right)=p_{k}(\mu)-\varepsilon$ и $\mu^{\varepsilon}\left(B\left(\gamma_{i}\right)\right)=p_{i}(\mu)$ при $i \neq k, m$. Тогда $h\left(\sigma_{f}, \mu_{f}^{\varepsilon}\right)>h\left(\sigma_{f}, \mu_{f}\right)=h\left(\sigma_{f}\right)$. Противоречие с определением величины $h\left(\sigma_{f}\right)$. Поэтому $p_{i}(\mu)>0$ при любом $i$.

Положим $p_{1}=1-\sum_{i=2}^{\infty} p_{i}$. Тогда выражение в фигурных скобках в (11) можно рассматривать как функцию

$$
\begin{aligned}
F\left(p_{2}, \ldots\right)= & -\sum_{i=2}^{\infty} p_{i}\left(h\left(\sigma_{f}\right) f^{*}\left(\gamma_{i}\right)+\ln p_{i}\right) \\
& -\left(1-\sum_{i=2}^{\infty} p_{i}\right) \ln \left(1-\sum_{i=2}^{\infty} p_{i}\right)-h\left(\sigma_{f}\right) f^{*}\left(\gamma_{1}\right)\left(1-\sum_{i=2}^{\infty} p_{i}\right)
\end{aligned}
$$

от переменных $p_{2}, p_{3}, \ldots$, где $p_{i} \geqslant 0$ при $i \geqslant 2, \sum_{i=2}^{\infty} p_{i} \leqslant 1$ и $\sum_{i=2}^{\infty} f^{*}\left(\gamma_{i}\right) p_{i}$ $<\infty$. Как мы убедились выше, все эти неравенства являются строгими, когда $p_{i}=p_{i}(\mu), i \geqslant 2$. Таким образом, функция $F$ дифференцируема относительно каждого переменного $p_{i}, i \geqslant 2$, в точке $\left\{p_{i}(\mu)\right\}$ и, кроме того, в этой точке

$$
\partial F / \partial p_{i}=0, \quad i \geqslant 2 .
$$

Последнее равенство дает следующие соотношения при $i \geqslant 2$ :

$$
\ln p_{i}(\mu)+h\left(\sigma_{f}\right) f^{*}\left(\gamma_{i}\right)-\ln \left(1-\sum_{j=2}^{\infty} p_{j}(\mu)\right)-h\left(\sigma_{f}\right) f^{*}\left(\gamma_{1}\right)=0 .
$$


Отсюда получаем, что $p_{i}(\mu)=c_{1} \exp \left(-h\left(\sigma_{f}\right) f^{*}\left(\gamma_{i}\right)\right)$ при $i \geqslant 1$, где $c_{1}=$ $p_{1}(\mu) \exp \left(h\left(\sigma_{f}\right) f^{*}\left(\gamma_{1}\right)\right)$. Из $(11)$ следует равенство $-\ln c_{1}=0$. Поэтому $c_{1}=1$. Так как $\left\{p_{i}(\mu)\right\}$ - вероятностный вектор, то справедливо (9). Из условия $\int f d \mu<\infty$ получаем неравенство (10).

Равенства

$$
\mu(B(\gamma))=\exp \left(-h\left(\sigma_{f}\right) f^{*}(\gamma)\right) \quad \text { при } \gamma \in K(G ; \mathbf{v})
$$

однозначно определяют меру $\mu$ в классе $\hat{I}_{f}^{\infty}(X(G))$. Очевидно, что $0<$ $\mu(B(\mathbf{w}))<\infty$ для любого $\mathbf{w} \in V(G)$. Повторяя приведенные выше рассуждения, получим, что условия (9) и (10) справедливы при любом w $\in V(G)$ и мера $\mu^{\prime}$, для которой $\mu^{\prime}(B(\mathbf{w}))=1$ и $\mu_{f}^{\prime} \in \hat{I}\left(X_{f}(G) ; 0\right)$, единственна и, следовательно, с точностью до постоянного множителя совпадает с $\mu$.

Пусть теперь выполнены условия (9) и (10). Тогда мера $\mu$, определенная при помощи формулы $(12)$ и условия $\mu\left(X(G) \backslash B^{\infty}(\mathbf{v})\right)=0$, принадлежит $\hat{I}_{f}^{\infty}(X(G))$ и

$$
h(\sigma, \mu)-h\left(\sigma_{f}\right) \int f d \mu=-\sum_{\gamma \in L(G ; \mathbf{v})} \mu(B(\gamma))\left(h\left(\sigma_{f}\right) f^{*}(\gamma)+\ln \mu(B(\gamma))\right)=0 .
$$

Поэтому $h\left(\sigma_{f}, \mu_{f}\right)=h\left(\sigma_{f}\right)$. Теорема доказана.

Используя вычисления из работы [19] (см. п. 3.10), нетрудно проверить, что мера $\mu$, для которой $\mu_{f} \in \hat{I}\left(X_{f}(G) ; 0\right)$, совпадает с распределением вероятностей, отвечающим некоторой стационарной цепи Маркова (ее переходные вероятности см. в (3.30) и (3.35) [19]). Если $\sum_{\gamma \in L(G ; \mathbf{v})} \ell(\gamma) \exp \left(-h\left(\sigma_{f}\right) f^{*}(\gamma)\right)$ $<\infty$, то эта цепь Маркова является положительно рекуррентной и мера $\mu$ с точностью до множителя совпадает с единственным элементом множества $\hat{I}\left(X(G) ; h\left(\sigma_{f}\right) f\right)$. В противном случае $\hat{I}\left(X(G) ; h\left(\sigma_{f}\right) f\right)=\varnothing$ и соответствующая цепь Маркова является нуль-рекуррентной.

Для любого $\mathbf{v} \in V(G)$ положим

$$
\delta_{\mathbf{v}}(f)=\sup \left\{\varepsilon \geqslant 0: \sum_{\gamma \in L(G ; \mathbf{v})} \exp \left(-h\left(\sigma_{f}\right)(1-\varepsilon) f^{*}(\gamma)\right)<\infty\right\}
$$

СЛЕДСТВИЕ 1. Предположим, что $\delta_{\mathbf{v}}(f)>0$ при некотором (а следовательно, и при любом; см. лемму 13 из [11]) $\mathbf{v} \in V(G)$. Тогда найдется такое $\varepsilon>0$, ито для потока $\sigma_{f+g}$, где $g$ - любая неотричательная функиия из класса $B^{0}(X(G))$ c $\|g\|=\sup _{x \in X(G)}|g(x)|<\varepsilon$, существует (и единственна) мера с максимальной энтропией.

ДокАЗАТЕльство. В силу теоремы 3 при $\delta_{\mathbf{v}}(f)>0$ мера с максимальной энтропией для потока $\sigma_{f}$ существует (и единственна). Используя выражение (8) для величины $h(f)$, убеждаемся, что это условие равносильно неравенству $h\left(\sigma_{f+t \chi_{B(\mathbf{v})}}\right)<h\left(\sigma_{f}\right)$, где $\chi_{B(\mathbf{v})}$ - характеристическая функция множества $B(\mathbf{v})$, при некотором (а следовательно, и при любом) $t>0$. Положим $\beta=\left(h\left(\sigma_{f+t \chi_{B(\mathrm{v})}}\right)+h\left(\sigma_{f}\right)\right) / 2$ и $\varepsilon=P(X(G) ; \beta f) / \beta$. Очевидно, что 
$0<\varepsilon<\infty$ и $h\left(\sigma_{f+\varepsilon}\right)=\beta$. Для любого $g \in B^{0}(X(G))$, удовлетворяющего неравенству $0 \leqslant g(x) \leqslant \varepsilon$ при каждом $x \in X(G)$, имеем

$$
h\left(\sigma_{f+g+t \chi_{B(\mathbf{v})}}\right) \leqslant h\left(\sigma_{f+t \chi_{B(\mathbf{v})}}\right), \quad h\left(\sigma_{f+g}\right) \geqslant h\left(\sigma_{f+\varepsilon}\right)=\beta .
$$

Поэтому $h\left(\sigma_{f+g+t \chi_{B(\mathbf{v})}}\right)<h\left(\sigma_{f+g}\right)$. Следствие доказано.

Из следствия 1 видно, что неравенство $\delta_{\mathbf{v}}(f)>0$ играет точно такую же роль, как и неравенство $\mathbf{r}_{\mathbf{v}}(f)>\exp (-P(X(G) ; f))$ в случае дискретного времени (см. $\S \S 4,5$ из [11]). В $\S 6$ работы [11] при этом условии и некоторых ограничениях на геометрию связного графа $G$ построено мероморфное продолжение дзета-функции Рюэля-Смейла (определение см. в $\S 4$ данной работы) и получен закон распределения числа периодических траекторий, аналогичный закону Чебышёва распределения простых чисел.

\section{§4. Специальные потоки, построенные по конечным ТМЦ и неотрицательным функциям из класса $\mathscr{F}(X(G) ; \mathbf{v})$}

Пусть $\mathbf{v}$ - некоторый фиксированный элемент множества $V(G)$. Обозначим через $\mathscr{F}(X(G) ; \mathbf{v})$ класс непрерывных функций, постоянных на цилиндрических множествах вида $\sigma^{k} B(\gamma)$, где $\gamma \in L(G ; \mathbf{v}), 0 \leqslant k \leqslant \ell(\gamma)-1$.

1. Меры с максимальной энтропией. Случай $h\left(\sigma_{f}\right)<\infty$. Пусть $G-$ полный граф с множеством вершин $V(G)=\{0 ; 1\}$. Через $\mathscr{F}_{+}(X(G) ; 1)$ обозначим совокупность неотрицательных функций $f$ из класса $\mathscr{F}(X(G) ; 1)$, для которых справедливо условие $(1)$ и $J(f)=\{\tilde{x}\}$, где $\tilde{x}$ - точка пространства $X(G)$ с координатами, равными 0 . Если $f \in \mathscr{F}_{+}(X(G) ; 1)$, то любая мера из множества $\hat{I}\left(X_{f}(G)\right)$ или сосредоточена в неподвижной точке $(\tilde{x}, 0)$, или имеет вид $\mu_{f}$, где $\mu \in \hat{I}_{f}^{\infty}(X(G))$ и $0<\mu(B(1))<\infty$. Как и в случае $f \in B^{0}(X(G))$, значение интеграла функции $f \in \mathscr{F}(X(G) ; 1)$ по такой мере $\mu$ зависит только от ее значений на множествах $B(\gamma)$, где $\gamma \in L(G ; 1)$ (см. (4); для любой функции $f \in \mathscr{F}(X(G) ; \mathbf{v})$ величина $f^{*}(\gamma)$, где $\gamma \in K(G ; \mathbf{v})$, определяется точно так же, как это сделано в $\S 3$ (см. (3))). Повторяя рассуждения леммы 1 и теоремы 2 , отсюда получаем, что $h\left(\sigma_{f}\right)=h(f)$ и справедливо следующее утверждение.

Teорема 4. Пусть $f \in \mathscr{F}_{+}(X(G) ; 1)$ u $h\left(\sigma_{f}\right)<\infty$. Тогда мера с максимальной энтропией для потока $\sigma_{f}$ существует (и единственна), если и только если при $\mathbf{v}=1$ выполнень условия (9) и (10).

Явный вид меры $\mu$, для которой $\mu_{f} \in \hat{I}\left(X_{f}(G) ; 0\right)$, может быть получен при помощи (12). Однако, вообще говоря, она теперь не обязана быть марковской мерой.

При $k \in \mathbb{Z}^{+}$обозначим через $j_{X(G), B(\mathbf{v}), f}^{(k)}(s)$ сумму ряда

$$
\sum_{\gamma \in L(G ; \mathbf{v})}\left(-h\left(\sigma_{f}\right) f^{*}(\gamma)\right)^{k} \exp \left(-h\left(\sigma_{f}\right) s f^{*}(\gamma)\right)
$$

(по понятным причинам при $k=1$ вместо верхнего индекса (1) в дальнейшем мы будем ставить «штрих»). Пусть $\gamma_{n}$ - цикл из множества $L(G ; 1)$ 
длины $n$. Очевидно, что в нашем случае элемент множества $L(G ; 1)$ такими условиями определяется однозначно.

Пример 1. Для любого $x \in X(G)$ введем величину $r(x)=\inf \left\{|i|: x_{i}=1\right\}$ и определим функцию $f \in \mathscr{F}_{+}(X(G) ; 1)$, положив

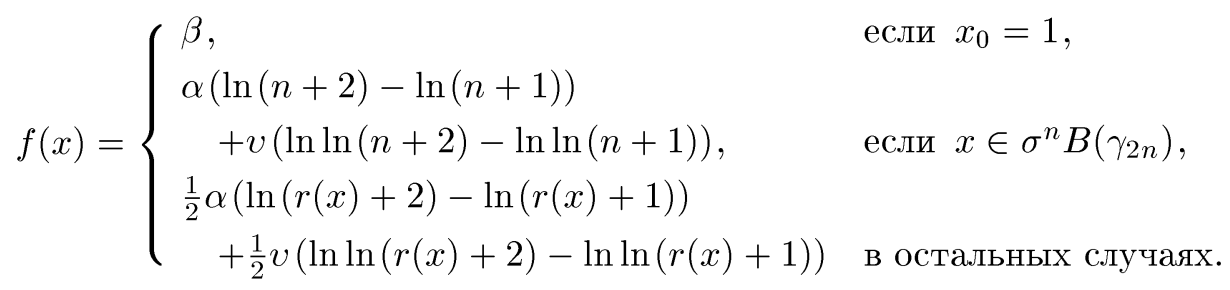

Нетрудно проверить, что

$$
f^{*}\left(\gamma_{n}\right)=\beta+\alpha(\ln ([n / 2]+2)-\ln 2)+v(\ln \ln ([n / 2]+2)-\ln \ln 2)
$$

при $n \geqslant 1$. Пусть $v=0$. При фиксированном $\alpha>1$ выберем $\beta>0$ так, чтобы $e^{-\beta}+2^{(\alpha+1)} e^{-\beta} \sum_{k=1}^{\infty}(k+2)^{-\alpha}=1$. Тогда $h\left(\sigma_{f}\right)=1$,

$$
j_{X(G), B(1), f}(s)=e^{-s \beta}+2^{\alpha s+1} e^{-s \beta} \sum_{k=1}^{\infty}(k+2)^{-\alpha s}
$$

и, следовательно, $\delta_{1}(f)>0$ (определение величины $\delta_{1}(f)$ см. в $(13)$ ). Поэтому существует мера с максимальной энтропией для потока $\sigma_{f}$ (очевидно, что для функции $f \in \mathscr{F}_{+}(X(G) ; 1)$ также справедливо утверждение следствия 1$)$. Если $\alpha \leqslant 2$, то эта мера имеет вид $\mu_{f}$, где $\mu(X(G))=\infty$. В противном случае можно считать, что $\mu \in \hat{I}\left(X(G) ; h\left(\sigma_{f}\right) f\right)$.

Пусть теперь $v>1$ и $\alpha=1$. Выберем $\beta>0$ так, чтобы

$$
e^{-\beta}+4(\ln 2)^{v} e^{-\beta} \sum_{n=1}^{\infty} \frac{1}{(n+2) \ln ^{v}(n+2)}<1 .
$$

Очевидно, что в этом случае $h\left(\sigma_{f}\right)=1$ и

$$
j_{X(G), B(1), f}(s)=e^{-s \beta}+2^{s+1}(\ln 2)^{s v} e^{-s \beta} \sum_{n=1}^{\infty}\left((n+2) \ln ^{v}(n+2)\right)^{-s} .
$$

Так как $j_{X(G), B(1), f}(1)<1$, то в силу теоремы 4 для потока $\sigma_{f}$ меры с максимальной энтропией не существует (при $v \leqslant 2$ справедливо равенство $j_{X(G), B(1), f}^{\prime}(1)=\infty$ и, следовательно, в данном случае можно выбрать $\beta$ так, чтобы $\left.j_{X(G), B(1), f}(1) \leqslant 1\right)$.

2. Законы распределения числа периодических траекторий. Случай $h\left(\sigma_{f}\right)<\infty$. В этом разделе мы исследуем законы распределения числа периодических траекторий $\tau$ в зависимости от их наименьших длин $\lambda(\tau)$. При $h\left(\sigma_{f}\right)<\infty$ положим

$$
\zeta_{\sigma_{f}}(s)=\prod_{\tau: \lambda(\tau) \neq 0}\left(1-\exp \left(-s h\left(\sigma_{f}\right) \lambda(\tau)\right)\right)^{-1}
$$


(здесь произведение берется по множеству $\Delta\left(\sigma_{f}\right)$ всех периодических траекторий потока $\sigma_{f}$ с $\lambda(\tau) \neq 0$ ). Теорема $3^{\prime}$ работы [13] позволяет получить следующее утверждение.

Tеорема 5. Пусть $f \in \mathscr{F}_{+}(X(G) ; 1) u h\left(\sigma_{f}\right)<\infty$. Тогда при Re $s>1$ справедливо тождество

$$
\zeta_{\sigma_{f}}(s)=\frac{1}{1-j_{X(G), B(1), f}(s)} .
$$

Из (15) следует, что дзета-функция Рюэля-Смейла $\zeta_{\sigma_{f}}(s)$ допускает мероморфное продолжение за пределы полуплоскости $\operatorname{Re} s>1$ тогда и только тогда, когда $\delta_{1}(f)>0$. Очевидно, что оно не обращается в нуль в полуплоскости $\operatorname{Re} s>1-\delta_{1}(f)$ и имеет простой полюс в точке $s=1$ (заметим, что $j_{X(G), B(1), f}(1)=1$ и $j_{X(G), B(1), f}^{\prime}(1)<0$ при $\left.\delta_{1}(f)>0\right)$. Если $\left\{\lambda(\tau): \tau \in \Delta\left(\sigma_{f}\right)\right\} \nsubseteq a \mathbb{Z}$ при любом $a \in \mathbb{R}$, то дзета-функция $\zeta_{\sigma_{f}}(s)$ больше полюсов на прямой $\operatorname{Re} s=1$ не имеет (простое доказательство этого факта см. в следствии 3 из [13]). Применяя тауберову теорему Икехары-Винера, отсюда нетрудно получить (см. [2]) следующий закон распределения числа периодических траекторий потока $\sigma_{f}$ :

$$
\operatorname{card}\{\tau: \lambda(\tau)<y\} \sim \frac{\exp \left(h\left(\sigma_{f}\right) y\right)}{h\left(\sigma_{f}\right) y} .
$$

Если $\delta_{1}(f)=0$, то закон (16) может не выполняться для соответствующего потока $\sigma_{f}$. Действительно, предположим, что $h\left(\sigma_{f}\right)=1, j_{X(G), B(1), f}(1)<1$ и $\left|j_{X(G), B(1), f}^{(k)}(1)\right|<\infty$ при некотором $k \geqslant 1$ (такую функцию можно получить, положив $v>k+1$ в примере 1). Тогда $\sum_{\tau \in \Delta\left(\sigma_{f}\right)} \lambda(\tau)^{k} \exp (-\lambda(\tau))<$ $\left|\zeta_{\sigma_{f}}^{(k)}(1) / \zeta_{\sigma_{f}}(1)\right|<\infty$ (см. (14) и $\left.(15)\right)$. Очевидно, что при $h\left(\sigma_{f}\right)<\infty$ существует лишь конечное число $K$ таких $\tau$, что $\lambda(\tau)<k$ (в противном случае $\zeta_{\sigma_{f}}(s)=\infty$ при любом положительном $s$, а это противоречит (14) и $\left.(15)\right)$. Пусть $d=\sum_{\tau: \lambda(\tau) \geqslant k} \lambda(\tau)^{k} \exp (-\lambda(\tau))$. Функция $y^{k} \exp (-y)$ убывает при $y \geqslant k$. Поэтому при любом $y>0$ справедливо неравенство

$$
(\operatorname{card}\{\tau: \lambda(\tau)<y\}-K) y^{k} \exp (-y)<d,
$$

и, следовательно, $\operatorname{card}\{\tau: \lambda(\tau)<y\}<d \exp (y) / y^{k}+K$. Очевидно, что если $k \geqslant 2$, то закон (16) не выполняется для потока $\sigma_{f}$.

\section{ЛИТЕРАТУРА}

1. Абрамов Л. М. Об энтропии потока. ДАН СССР, 128, № 5, 873-875 (1959).

2. Parry $W$., Pollicott $M$. Zeta functions and the periodic orbit structure of hyperbolic dynamics. Astérisque, 187-188 (1990).

3. Krengel $U$. Entropy of conservative transformations. Z. Wahrscheinlichkeitstheorie verw. Gebiete, 7, 161-181 (1967).

4. Смейл C. Дифференцируемые динамические системы. УМН, 25, вып. 1, 113185 (1970).

5. Bowen R., Ruelle D. The ergodic theory of Axiom A flows. Invent. Math., 29, 181-202 (1975). 
6. Бунимович Л. А., Синай Я. Г., Чернов Н. И. Марковские разбиения для двумерных гиперболических биллиардов. УМН, 45, вып. 3, 97-134 (1990).

7. Синай Я. Г. Введение в эргодическую теорию. Серия БСМ. Вып. 1, Фазис, M., 1996.

8. Ruelle D. Generalized zeta-functions for axiom A basic sets. Bull. Am. Mats. Soc., 82, 153-156 (1976).

9. Parry $W$., Pollicott $M$. An analogue of the prime number theorem and closed orbits of Axiom A flows. Annals of Math., 118, 573-591 (1983).

10. Huber $H$. Zur analytischen Theorie hyperbolischer Raum formen und Bewegungsgruppen, II. Math. Ann., 142, 385-398 (1961).

11. Савченко $C$. В. Периодические точки счетных топологических марковских цепей. Матем. сб., 186, № 10, 103-140 (1995).

12. Bowen R., Walters P. Expansive one-parameter flows. J. Differential Equations, 12, 180-193 (1972).

13. Савченко $C$. B. Равновесные состояния с неполными носителями и периодические траектории. Матем. заметки, 59, вып. 2, 230-253 (1996).

14. Parry $W$. Ergodic and spectral analysis of certain infinite measure preserving transformations. Proc. Am. Math. Soc., 16, 960-966 (1965).

15. Sucheston $L$. A note on conservative transformations and the recurrence theorem. Amer. J. Math., 79, 444-447 (1957).

16. Колмогоров А. Н. Новый метрический инвариант транзитивных динамических систем и автоморфизмов пространств Лебега.ДАН СССР, 119, № 5, 861-864 (1958).

17. Рохлин B. A. Лекции по энтропийной теории преобразований с инвариантной мерой. УМН, 22, вып. 5, 3-56 (1967).

18. Абрамов Л. М. Об энтропии производного автоморфизма. ДАН СССР, 128, № 4, 647-650 (1959).

19. Gurevich B. M. A variational characterization of one-dimensional countable state Gibbs random fields. Z. Wahrscheinlichkeitstheorie verw. Gebiete, 68, 205-242 (1984).

Институт теоретической физики РАН им. Л. Д. Ландау

Поступила в редакцию e-mail: savch@icp.ac.ru 